\title{
Poincaré invariance in low-energy EFTs for QCD
}

\author{
Sungmin Hwang ${ }^{1,2, a}$ \\ ${ }^{1}$ Physik-Department I, Technische Universität München, T30f \\ ${ }^{2}$ Max-Planck-Institut für Physik (Werner-Heisenberg-Institut), München
}

\begin{abstract}
We present the calculations on deriving constraints between the Wilson coefficients in non-relativistic quantum chromodynamics and potential non-relativistic quantum chromodynamics by exploiting the symmetry of its fundamental theory, Poincaré invariance in particular. Implications of the constraints are briefly discussed in the context of the effective string theory.
\end{abstract}

\section{Introduction}

During the last decades, quantum chromodynamics (QCD) has been verified theoretically and experimentally as the solid theory for explaining color interactions at subatomic scales. However, QCD contains both perturbative and non-perturbative aspects, which are manifested by the asymptotic freedom [1-3] and the confinement [4], respectively. While perturbative QCD (pQCD) [5] is the proper framework for describing interactions at high energies, it is not able to give any feasible description of its dynamics around the scale at which the confinement phase takes place. Thus, it is helpful to separate the perturbative and non-perturbative regimes.

Separately treating those two different aspects of physics calls upon a framework of effective field theories (EFTs), which requires to observe a hierarchy of scales in the physical systems of one's interest. In case of heavy mesons, such as the bottomonium or charmonium states, the hierarchy is given such that the rest mass of single quark/antiquark $M$ is much greater than the momentum transfer $M v$ between them ( $v$ being the relative velocity) and the confinement scale $\Lambda_{Q C D}: M \gg$ $M v, \Lambda_{Q C D}$. As the heavy scale $M$ is integrated out, the dynamics of the heavy quark fields enter into the non-relativistic regime, so that the resulting effective theories are either non-relativistic $Q C D$ (NRQCD) [6, 7] for heavy quark-antiquark systems or the heavy quark effective theory (HQET) [8, 9] for heavy quark and light quark states, whose Lagrangians is organized according to $1 / M$ expansion

$$
\mathcal{L}_{N R Q C D}=\sum_{n} c_{n} \frac{O_{n}}{M^{d_{n}-4}}
$$

where operators $O_{n}$ consist of effective degrees of freedom (DOFs) with its mass dimension $d_{n}$, and $c_{n}$ are the undetermined scalar functions, namely the Wilson (or matching) coefficients. These coefficients contain information of its underlying (or UV) theory, whose exact values are to be

\footnotetext{
ae-mail: sungmin.hwang@tum.de
} 
determined either via matching to the UV theory at the corresponding energy scale or by experiments.

NRQCD is the useful EFT for analysing productions (or annihilations) of the heavy quarkantiquark states, but it shows difficulties in explaining the mass splittings or transitions of the bound states. In order to resolve these issues, one can construct a valid EFT for such cases, which is the potential NRQCD (pNRQCD)[10]. When the hierarchy of scale is established as such, $M \gg M v \gg M v^{2},\left(M v^{2}\right.$ being the relative kinetic energy between quark and antiquark), pNRQCD is constructed by integrating out the relative momentum $M v$ from NRQCD, and its Lagrangian is organized by the multipole expansion in the relative distance $r$, in addition to the $1 / M$ expansion from NRQCD. Just like in the case of NRQCD/HQET, the pNRQCD Lagrangian contains a series of the effective operators along with the Wilson coefficients, and these coefficients are to be determined via matching to NRQCD, the underlying theory of pNRQCD. It is apparent that calculations can easily become complicated and lengthier if one tries to perform the matching directly from QCD to NRQCD and then to pNRQCD, due to the proliferation of the effective operators in the EFTs. It is, therefore, worthwhile to look for some ways to reduce the amount of the matching calculations, such as by deriving constraints between the coefficients.

There have been two separate ways to deriving constraints between the Wilson coefficients, both of which exploit symmetries of the EFTs. The first symmetry is the reparametrisation invariance [11], and the second is the Poincaré invariance [13, 14], which we explore in detail here. Since QCD is a Poincaré invariant theory ${ }^{1}$, it is natural to observe that its low-energy counterpart, NRQCD in particular, is also to observe Poincaré invariance ${ }^{2}$. As the Poincaré symmetry is realized in NRQCD, pNRQCD is also to preserve the same symmetry by the same logic, thereby constraints between the Wilson coefficients can be established as a result.

Concerning the derivations of the constraints, this proceeding paper is organized as the following: in Sec. 2, general principles of Poincaré transformation of non-relativistic fields are discussed, which we apply to NRQCD and derive the constraints between the coefficients. In Sec. 3, we present similar arguments on the pNRQCD, but some complications arise due not only to the proliferations of the effective operators, but also to the transformation behavior of the fields; these issues are addressed in the same section. Implications of the constraints in pNRQCD are briefly discussed in Sec. 4, with the framework of the effective string theory [15], which is the EFT for the flux tube model of the heavy quark-antiquark bound system at the long-distance limit.

\section{Poincaré invariance in NRQCD}

In relativistic field theories, its field transformation under the Poincaré group is given as

$$
\phi_{a}(x) \rightarrow \mathcal{M}_{a b}(\Lambda) \phi_{b}\left(\Lambda^{-1} x\right)
$$

where $\phi$ is the generic relativistic field with $\mathcal{M}_{a b}(\Lambda)$ being the finite dimensional representation of the Poincaré group and $\Lambda$ being the transformation matrix which acts on the coordinate $x^{\prime \mu}=\Lambda_{v}^{\mu} x^{\nu}$. There are ten independent components for the transformation matrix, three of which are responsible for the

\footnotetext{
${ }^{1}$ Poincaré symmetry is one of the two pillars upon which the quantum field theory is built along with the cluster decomposition principle [12].

${ }^{2}$ Although the fields in the EFT Lagrangian are non-relativistic (it will be clarified in the next section), the Lagrangian itself can observe Poincaré invariance order by order in the expansion parameter.
} 
spatial rotations, three are for the spatial boosts, and the rest four of them are the spacetime translations. Since only the spatial boosts from the representation of the Poincaré group is no longer linear at the non-relativistic regime, let us pay our attention to such case. Then the boost transformation of a (free) relativistic field is given by

$$
\phi_{a}(x) \rightarrow\left(e^{\mp \eta \cdot \mathbf{\Sigma}}\right)_{a b} \phi_{b}\left(\mathcal{B}^{-1} x\right),
$$

in which $\eta$ is the parameter for the boost, $\Sigma$ is the spin representation, and $\mathcal{B}$ is the boost transformation matrix that acts on spacetime coordinates. In case of a massive field with mass $M$, one can rewrite the boost transformation in a slightly different manner when the rest frame of the massive particle, $v=(1,0,0,0)$, is chosen $[14]$

$$
\phi_{a}(x) \rightarrow \exp \left[\mp \boldsymbol{\eta} \cdot \frac{\boldsymbol{\Sigma} \times \boldsymbol{\partial}}{M+\sqrt{M^{2}-\boldsymbol{\partial}^{2}}}\right]_{a b} \phi_{b}\left(\mathcal{B}^{-1} x\right) .
$$

The boost transformation has now changed from global to local (appearance partial derivatives), and one can take a non-relativistic expansion of this expression in order to apply to the non-relativisitic field theory. For an interacting case, the boost transformation of the field with regard to symmetries under charge, parity and time reversal, $(C, P, T$ in short), as well as spatial rotations, are given by (up to a cubic order in $1 / M)[16]$

$$
\phi_{a}(x) \rightarrow\left\{1+i M \boldsymbol{\eta} \cdot \boldsymbol{x}-\frac{i k_{1} \boldsymbol{\eta} \cdot \boldsymbol{D}}{2 M}+\frac{k_{2}(\boldsymbol{\Sigma} \times \boldsymbol{\eta}) \cdot \boldsymbol{D}}{2 M}-\frac{i k_{3} \boldsymbol{\eta} \cdot \boldsymbol{D} \boldsymbol{D}^{2}}{4 M^{3}}+\frac{k_{4}(\boldsymbol{\Sigma} \times \boldsymbol{\eta}) \cdot \boldsymbol{D}}{2 M} \frac{\boldsymbol{D}^{2}}{4 M^{2}}+O\left(g, 1 / M^{4}\right)\right\} \phi_{a}\left(\mathcal{B}^{-1} x\right),
$$

in which $k$ 's are the undetermined coefficients. It is clear that $\boldsymbol{D}$ is the gauge covariant derivatives for the interacting theory. Let us now apply this expression to NRQCD whose bilinear parts of the Lagrangian in the heavy quark and antiquark fields are given by $[6,7]$

$$
\begin{aligned}
\mathcal{L}_{\mathrm{NRQCD}} & \ni \psi^{\dagger}\left\{i D_{0}+c_{1} \frac{\boldsymbol{D}^{2}}{2 M}+c_{2} \frac{\boldsymbol{D}^{4}}{8 M^{3}}+c_{F} g \frac{\boldsymbol{\sigma} \cdot \boldsymbol{B}}{2 M}+c_{D} g \frac{[\boldsymbol{D} \cdot \boldsymbol{E}]}{8 M^{2}}+i c_{s} g \frac{\boldsymbol{\sigma} \cdot[\boldsymbol{D} \times, \boldsymbol{E}]}{8 M^{2}}\right\} \psi \\
& +\chi^{\dagger}\left\{i D_{0}-c_{1} \frac{\boldsymbol{D}^{2}}{2 M}-c_{2} \frac{\boldsymbol{D}^{4}}{8 M^{3}}-c_{F} g \frac{\boldsymbol{\sigma} \cdot \boldsymbol{B}}{2 M}+c_{D} g \frac{[\boldsymbol{D} \cdot, \boldsymbol{E}]}{8 M^{2}}+i c_{s} g \frac{\boldsymbol{\sigma} \cdot[\boldsymbol{D} \times, \boldsymbol{E}]}{8 M^{2}}\right\} \chi
\end{aligned}
$$

where $c$ 's are the Wilson coefficients, $\psi$ annihilates the heavy quark, and $\chi$ creates the heavy antiquark. From Eq. (5), these non-relativistic quark and antiquark fields transform under the boost as (up to the linear order in $1 / M$ )

$$
\begin{aligned}
& \psi(x) \rightarrow\left\{1+i M \boldsymbol{\eta} \cdot \boldsymbol{x}-\frac{i k_{1}}{2 M} \boldsymbol{\eta} \cdot \boldsymbol{D}+\frac{k_{2}}{4 M} \boldsymbol{\eta} \cdot(\boldsymbol{D} \times \boldsymbol{\sigma})+O\left(g, M^{-2}\right)\right\} \psi\left(\mathcal{B}^{-1} x\right) \\
& \chi(x) \rightarrow\left\{1-i M \boldsymbol{\eta} \cdot \boldsymbol{x}+\frac{i k_{1}}{2 M} \boldsymbol{\eta} \cdot \boldsymbol{D}-\frac{k_{2}}{4 M} \boldsymbol{\eta} \cdot(\boldsymbol{D} \times \boldsymbol{\sigma})+O\left(g, M^{-2}\right)\right\} \chi\left(\mathcal{B}^{-1} x\right)
\end{aligned}
$$

and applying Eq. (7) and Eq. (8) to Eq. (6), we obtain the original form of the bilinear sectors of the NRQCD Lagrangian and additional terms up to total derivatives (also up to the linear order in $1 / M$ )

$$
\delta \mathcal{L}_{2 \psi}=\psi^{\dagger}\left[i\left(1-c_{1}\right) \boldsymbol{\eta} \cdot \boldsymbol{D}-\frac{1}{2 M}\left(k_{1}-c_{1}\right)\left\{D_{0}, \boldsymbol{\eta} \cdot \boldsymbol{D}\right\}+\frac{1}{4 M}\left(1-2 c_{F}+c_{s}\right) \boldsymbol{\eta} \cdot(g \boldsymbol{E} \times \boldsymbol{\sigma})\right] \psi .
$$

This expression is to vanish up to total derivatives, according to the Poincaré invariance, which implies $k_{1}=c_{1}=1, c_{s}=2 c_{F}-1$. Note that the coefficient $k_{2}$ from the boost transformations, Eq. (7) and Eq. (8), is still to be determined. It can be fixed by the commutation relations between boost generators which is to be equivalent to the rotation generator up to $1 / M$ order; it turns out that $k_{2}$ equals to the unity. These results confirm the values from the literature [13], but the amount of calculations involved 
here is smaller and simpler than the hitherto known methods, which means that one can apply it for the higher loop calculations with much ease. Similar procedures are applied to the four-quark sector of NRQCD, and the results are in accord with known values [17]; several new features are also to be shown. Full results on the four-quark sector will be discussed in the upcoming paper.

\section{Poincaré invariance in pNRQCD}

pNRQCD Lagrangian is obtained by integrating out the momentum transfer $M v \sim 1 / r$ from NRQCD [10], thereby a slight change in its coordinate system arises: spatial dependence of the color singlet and octet fields is on the centre-of-mass $\boldsymbol{R}=\left(\boldsymbol{x}_{1}+\boldsymbol{x}_{2}\right) / 2$ as well as on the relative coordinates $\boldsymbol{r}=\boldsymbol{x}_{1}-\boldsymbol{x}_{2}$, where $\boldsymbol{x}_{1}$ and $\boldsymbol{x}_{2}$ are the spatial coordinates of the heavy quark and the heavy antiquark, respectively. Also the ultrasoft gluons have spatial dependence only on the center of mass coordinate; in short, the non-relativistic DOFs in pNRQCD are the singlet $S(t, \boldsymbol{R}, \boldsymbol{r})$ and the octet fields $O^{a}(t, \boldsymbol{R}, \boldsymbol{r})$, with $a$ being the color index, and we denote the gluons with $A_{\mu}^{a}(t, \boldsymbol{R})$. Power counting schemes are followed $\nabla_{r}, 1 / r \sim M v, \partial_{0}, \nabla_{R}, A_{\mu} \sim M v^{2}, \boldsymbol{E}, \boldsymbol{B} \sim M^{2} v^{4}$, so that the schematic form of the pNRQCD Lagrangian bilinear in singlet and octet fields includes

$$
\mathcal{L}_{p N R Q C D} \ni \int d^{3} r \operatorname{Tr}\left\{S^{\dagger} \mathcal{K}_{S S} S+O^{\dagger} \mathcal{K}_{O O} O+\left[S^{\dagger} \mathcal{K}_{S O} O+\text { H.C. }\right]\right\}-\frac{1}{4} F_{\mu v}^{a} F^{\mu v, a}(t, \mathbf{R})
$$

Note that there is a integration over the relative distance, which is due to the fact that the multipole expansion is in place. H.C. includes hermitian conjugate terms so that the expressions inside the trace is hermitian in total and $\mathcal{K}$ operators contain charge conjugate terms, which means that the terms inside the trace are charge neutral in total. As for the simplicity of the argument in this paper, we only focus on the first term inside the trace, which is the bilinear parts of the singlet field (up to $1 / M^{2}$ ) [13]

$$
\begin{aligned}
S^{\dagger} \mathcal{K}_{S S} S= & S^{\dagger}\left(i \partial_{0}+\frac{1}{2 M}\left\{c_{s}^{(1,-2)}, \nabla_{r}^{2}\right\}+\frac{c_{s}^{(1,0)}}{4 M} \nabla_{R}^{2}-V_{S}^{(0)}-\frac{V_{S}^{(1)}}{M}+\frac{V_{p^{2} S a}}{8 M^{2}} \nabla_{R}^{2}+\frac{1}{2 M^{2}}\left\{\nabla_{r}^{2}, V_{p^{2} S b}\right\}\right. \\
& +\frac{V_{L^{2} S a}}{4 M^{2} r^{2}}\left(\boldsymbol{r} \times \nabla_{R}\right)^{2}+\frac{V_{L^{2} S b}}{4 M^{2} r^{2}}\left(\boldsymbol{r} \times \nabla_{r}\right)^{2}-\frac{V_{S_{12} S}}{M^{2} r^{2}}\left(3\left(\boldsymbol{r} \cdot \boldsymbol{\sigma}^{(1)}\right)\left(\boldsymbol{r} \cdot \boldsymbol{\sigma}^{(2)}\right)-\boldsymbol{r}^{2} \boldsymbol{\sigma}^{(1)} \cdot \boldsymbol{\sigma}^{(2)}\right) \\
& \left.-\frac{V_{S^{2} S}}{4 M^{2}} \boldsymbol{\sigma}^{(1)} \cdot \boldsymbol{\sigma}^{(2)}+\frac{i V_{L S S a}}{4 M^{2}}\left(\boldsymbol{r} \times \nabla_{R}\right) \cdot\left(\boldsymbol{\sigma}^{(1)}-\boldsymbol{\sigma}^{(2)}\right)+\frac{V_{L S S b}}{4 M^{2}}\left(\boldsymbol{r} \times \boldsymbol{\nabla}_{r}\right) \cdot\left(\boldsymbol{\sigma}^{(1)}+\boldsymbol{\sigma}^{(2)}\right)\right) S .
\end{aligned}
$$

Note that $c$ 's and $V$ 's are the undetermined Wilson coefficients; superscripts on $c$ 's denote the orders of $1 / M$ and $r$, respectively, and $V$ 's are the potential terms. Also, the superscript to the Pauli matrices $\sigma^{(1 / 2)}$ denotes the spin projection for the heavy quark/antiquark, respectively. Our task here is to construct the most general form of the spatial boost which acts on the singlet field with the symmetries under $C, P, T$ transformations in mind

$$
\mathbf{k} \stackrel{P}{\rightarrow}-\mathbf{k}, \quad \mathbf{k} \stackrel{C}{\rightarrow}-\sigma_{2} \mathbf{k}^{*} \sigma_{2}, \quad \mathbf{k} \stackrel{T}{\rightarrow} \sigma_{2} \mathbf{k} \sigma_{2}
$$


Then up to the linear order of $1 / M$ and $r$, the boost transformation of the singlet field is shown explicitly as [16]

$$
\begin{aligned}
& S^{\prime}(t, \boldsymbol{R}, \boldsymbol{r})=\left(1-2 i M \boldsymbol{\eta} \cdot \boldsymbol{R}+\frac{i k_{D}^{(1,0)}}{4 M} \boldsymbol{\eta} \cdot \boldsymbol{\nabla}_{R}+\frac{i}{4 M}\left\{k_{a^{\prime}}^{(1,0)} \boldsymbol{\eta} \cdot \boldsymbol{r}, \boldsymbol{\nabla}_{R} \cdot \boldsymbol{\nabla}_{r}\right\}\right. \\
& +\frac{i}{4 M}\left\{k_{a^{\prime \prime}}^{(1,0)} \boldsymbol{r} \cdot \boldsymbol{\nabla}_{R}, \boldsymbol{\eta} \cdot \boldsymbol{\nabla}_{r}\right\}+\frac{i}{4 M}\left\{k_{a^{\prime \prime \prime}}^{(1,0)} \boldsymbol{r} \cdot \boldsymbol{\nabla}_{r}\left(\boldsymbol{\eta} \cdot \boldsymbol{\nabla}_{R}\right)\right\}+\frac{i}{4 M}\left\{\frac{k_{b}^{(1,0)}}{r^{2}}(\boldsymbol{\eta} \cdot \boldsymbol{r})\left(\boldsymbol{r} \cdot \boldsymbol{\nabla}_{R}\right) \boldsymbol{r} \cdot \boldsymbol{\nabla}_{r}\right\} \\
& -\frac{k_{c}^{(1,0)}}{8 M} \boldsymbol{\eta} \cdot \nabla_{R} \times\left(\sigma^{(1)}+\sigma^{(2)}\right)+\frac{k_{d^{\prime}}^{(1,0)}}{8 M r^{2}}\left(\boldsymbol{\eta} \cdot \boldsymbol{r} \times \boldsymbol{\nabla}_{R}\right)\left(\boldsymbol{r} \cdot\left(\boldsymbol{\sigma}^{(1)}+\boldsymbol{\sigma}^{(2)}\right)\right) \\
& -\frac{k_{d^{\prime \prime}}^{(1,0)}}{8 M r^{2}}\left(\boldsymbol{\eta} \cdot \boldsymbol{r} \times\left(\boldsymbol{\sigma}^{(1)}+\boldsymbol{\sigma}^{(2)}\right)\right)\left(\boldsymbol{r} \cdot \boldsymbol{\nabla}_{R}\right)-\frac{1}{8 M}\left\{k_{a}^{(1,-1)}, \boldsymbol{\eta} \cdot \nabla_{r} \times\left(\boldsymbol{\sigma}^{(1)}-\boldsymbol{\sigma}^{(2)}\right)\right\} \\
& +\frac{1}{8 M}\left\{\frac{k_{b^{\prime}}^{(1,-1)}}{r^{2}}\left(\boldsymbol{r} \cdot\left(\boldsymbol{\sigma}^{(1)}-\boldsymbol{\sigma}^{(2)}\right)\right)(\boldsymbol{\eta} \times \boldsymbol{r}) \cdot, \boldsymbol{\nabla}_{r}\right\} \\
& \left.-\frac{1}{8 M}\left\{\frac{k_{b^{\prime \prime}}^{(1,-1)}}{r^{2}}\left(\boldsymbol{\eta} \cdot \boldsymbol{r} \times\left(\boldsymbol{\sigma}^{(1)}-\boldsymbol{\sigma}^{(2)}\right)\right) \boldsymbol{r} \cdot \boldsymbol{\nabla}_{r}\right\}\right) S\left(t^{\prime}, \boldsymbol{R}^{\prime}, \boldsymbol{r}^{\prime}\right) \text {. }
\end{aligned}
$$

At a first glance, more problems seem to have arisen due to the emergence of the unknown coefficients $k$ 's in the boost transformation. Eq. (13) can, however, be simplified by the means of the field redefinitions, unitary transformations in particular: $S=\mathcal{U}_{S} \tilde{S}$ where $\tilde{S}$ is the newly defined singlet field and $\mathcal{U}_{S}$ is the unitary operator for it. A natural choice for the unitary operator is an exponential function with anti-hermitian operators for its exponents. A quadratic order of $1 / M$ expansion for the exponents comes from the boost transformation of the new singlet field, so that

$$
\mathcal{U}_{S}=\exp \left[-\frac{1}{4 M^{2}}\left\{q_{a^{\prime}}^{(1,0)} \boldsymbol{r} \cdot \boldsymbol{\nabla}_{R}, \boldsymbol{\nabla}_{r} \cdot \boldsymbol{\nabla}_{R}\right\}-\frac{1}{4 M^{2}}\left\{q_{a^{\prime \prime}}^{(1,0)} \boldsymbol{r} \cdot \boldsymbol{\nabla}_{R}, \boldsymbol{\nabla}_{r} \cdot \boldsymbol{\nabla}_{R}\right\}+\cdots\right],
$$

where the $q$ 's are the parameters which can freely be chosen. As we apply the unitary transformation to the singlet field, the boost coefficients are shifted like

$$
\begin{aligned}
& k_{a^{\prime}}^{(1,0)} \rightarrow k_{a^{\prime}}^{(1,0)}-2 q_{a^{\prime}}^{(1,0)}-2 q_{a^{\prime \prime}}^{(1,0)} \\
& k_{a^{\prime \prime}}^{(1,0)} \rightarrow k_{a^{\prime \prime}}^{(1,0)}-2 q_{a^{\prime \prime}}^{(1,0)}-2 q_{a^{\prime}}^{(1,0)} \\
& k_{a^{\prime \prime \prime}}^{(1,0)} \rightarrow k_{a^{\prime \prime \prime}}^{(1,0)}-4 q_{a^{\prime \prime \prime}}^{(1,0)} \\
& \ldots
\end{aligned}
$$

and $q$ 's chosen so that they eliminate as many terms in the boost as possible, thereafter only four boost coefficients, $k_{D}^{(1,0)}, k_{a^{\prime}}^{(1,0)}, k_{c}^{(1,0)}, k_{d^{\prime \prime}}^{(1,0)}$ remain. As was shown in the case of NRQCD, there exist additional constraints one can impose on the boost generator. First of which is the commutation relations between the boost generators acting onto the singlet field

$$
[1-i \boldsymbol{\xi} \cdot \boldsymbol{k}, 1-i \boldsymbol{\eta} \cdot \boldsymbol{k}] S=i(\boldsymbol{\xi} \times \boldsymbol{\eta}) \cdot \boldsymbol{j} S
$$

and it fixes three coefficients: $k_{a^{\prime}}^{(1,0)}=k_{c}^{(1,0)}=1, k_{d^{\prime \prime}}^{(1,0)}=0$. The last remaining one $k_{D}^{(1,0)}$ is fixed in the end along with deriving the constraints between the Wilson coefficients. The boost transformation of the singlet bilinear part of the pNRQCD Lagrangian is obtained by the simplified expression of the boost generator, thereby the original structure of the Lagrangian is kept intact up to total derivatives 
(organized up to the linear order in $1 / M$ )

$$
\begin{aligned}
\delta \mathcal{L}_{2 S} & =S^{\dagger}\left(i\left(1-c_{S}^{(1,0)}\right) \boldsymbol{\eta} \cdot \nabla_{R}-\frac{1}{2 M}\left(k_{D}^{(1,0)}-c_{S}^{(1,0)}\right) \boldsymbol{\eta} \cdot \nabla_{R} \partial_{0}-\frac{i}{M}\left(V_{p^{2} S a}+V_{L^{2} S a}+\frac{1}{2} V_{S}^{(0)}\right) \boldsymbol{\eta} \cdot \nabla_{R}\right. \\
& \left.+\frac{i}{M r^{2}}\left(V_{L^{2} S a}+\frac{r}{2} \partial_{r} V_{S}^{(0)}\right)(\boldsymbol{\eta} \cdot \boldsymbol{r})\left(\boldsymbol{r} \cdot \nabla_{R}\right)+\frac{1}{2 M}\left(V_{L S S a}+\frac{1}{2 r} \partial_{r} V_{S}^{(0)}\right) \boldsymbol{\eta} \cdot\left(\boldsymbol{\sigma}^{(1)}-\boldsymbol{\sigma}^{(2)}\right) \times \boldsymbol{r}\right) S
\end{aligned}
$$

which implies that all of the expressions inside the bracket in Eq. (17) are to vanish. Similar procedures are applied to the octet bilinear as well as to the singlet-octet sectors, and they also yield relations between the coefficients. Significance of this procedure is that the form of the boost generator we started with is greatly simplified and eventually coincides with the one inspired by Wigner's little group formalism $[14,18]$, due to a particular choice of the unitary transformation.

\section{Outlook: effective string theory}

Poincaré invariance in low-energy EFTs for QCD sheds light on an another effective description of the heavy quark-antiquark bound system, namely the effective string theory (EST) [15]. The EST is valid at the long-distance separation between the heavy quark and the heavy antiquark, which is below the confinement scale, and the transversal excitations of the long string with its two ends fixed are the DOFs. There has been a hypothesis that Wilson loop in NRQCD is related to the EST at long distance as well as at large time scale [19]. On the other hand, Wilson loop in NRQCD at large time are related to the static potential in pNRQCD by the matching calculation [20], which implies that one can relate the heavy quark-antiquark potentials to the string field variables. There arise dimensionful parameters in the string description [21], and Poincaré invariance in pNRQCD induces several constraints between the parameters in the EST [22]. In [22], these constraints are derived, but string variables are only considered up to the leading order, which are subjected to be modified when subleading terms are included; it is clear that coefficients to the several potential terms are to be altered, which is a part of our work in progress. Full EFT systematics to the EST concerning the inclusion of the subleading terms is to be discussed in the other proceeding paper from this conference [23] as well as in the follow-up paper to be appeared.

\section{References}

[1] D. Gross, F. Wilczek, Phys. Rev. Lett. 30, 1343 (1973)

[2] D. Gross, F. Wilczek, Phys. Rev. D 8, 3633 (1973)

[3] H. Politzer, Phys. Rev. Lett. 30, 1346 (1973)

[4] K. Wilson, Phys. Rev. D 10, 2445 (1974)

[5] J. Collins, Foundations of perturbative QCD (Cambridge: Cambridge University Press, 2013)

[6] W. Caswell, G. Lepage, Phys. Lett. B 167, 437-442 (1986)

[7] G. Bodwin, E. Braaten, G. Lepage, Phys. Rev. D 51, 1125 (1995)

[8] N. Isgur, M. Wise, Phys. Lett. B 232, 113 (1989)

[9] E. Eichten, B. Hill, Phys. Lett. B 234, 511 (1990)

[10] N. Brambilla, A. Pineda, J. Soto, A. Vairo, Nucl. Phys. B 566, 275-310 (2000)

[11] M. Luke, A. Manohar, Phys. Lett. B 286, 348-354 (1992)

[12] S. Weinberg, Quantum theory of fields I (Cambridge: Cambridge University Press, 1995)

[13] Brambilla N. Gromes D. and Vairo A. 2003 Phys. Lett. B 576, 314-327

[14] J. Heinonen, R. Hill, M. Solon, Phys. Rev. D 86, 094020 (2012) 
[15] J. Kogut, G. Parisi, Phys. Rev. Lett. 47, 16 (1981)

[16] M. Berwein, N. Brambilla, S. Hwang, A. Vairo, TUM-EFT 74/15 (2016)

[17] N. Brambilla, A. Vairo, E. Mereghetti, Phys. Rev. D 83, 079904 (2009)

[18] E. Wigner, Annals Math. 40, 149 (1939)

[19] M. Lüscher, Nucl. Phys. B 180, 317 (1981)

[20] N. Brambilla, A. Pineda, J. Soto, A. Vairo, Phys. Rev. D 63, 014023 (2000)

[21] G. Perez-Nadal, J. Soto, Phys. Rev. D 79, 114002 (2009)

[22] N. Brambilla, M. Groher, H. Martinez, A. Vairo, Phys. Rev. D 90, 114032 (2014)

[23] S. Hwang, Proc. 12th ed. of Confinement, Thessaloniki (2016) 